\title{
Un Modelo de Medición de Resultados en las Prácticas de Innovación Abierta
}

\section{A Model to Measure Results in Open Innovation Practices}

\author{
Carmen de Pablos-Heredero', Ignacio Soret-Los Santos², Máxima Juliana López-Eguilaz ${ }^{3}$
}

\begin{abstract}
El objetivo principal de este trabajo consiste en la propuesta de un modelo que permita la medición de conocimiento y la generación de eficiencias organizativas en las iniciativas de innovación abierta con los clientes. Además hemos tratado de analizar otro conjunto de aspectos necesarios para la propuesta del modelo: la identificación, selección y validación de índices que midan capital relacional y eficiencias organizativas. El modelo se compone de dos partes: una que trata de medir el capital relacional, y otra que mide la generación de eficiencias organizativas. El modelo generado es un modelo global que se puede aplicar en cualquier sector y en el que los clientes son el centro de los procesos de innovación donde los diferentes agentes interactúan. Deciden como se comportarán los usos de los nuevos productos y servicios.
\end{abstract}

Keywords: aproximación lead-user; estrategias de colaboración; capital relacional; eficiencias organizativas.

The main objective of this work consists of proposing a model that will allow measuring knowledge and the generation of organizational efficiencies in open innovation initiatives with customers. Besides we have tried to analyze another group of aspects needed to propose the model: the identification, selection and validation of indexes that measure the relational capital and organizational efficiencies. The model is composed by two parts: one in which we try to measure relational capital, and another one that measures the generation of organizational efficiencies. The model offered is a global one that can be applied to any industry where the customers are the centre of the innovation processes where the different agents interact. They decide how future uses of new products and services will take place.

Keywords: lead-user approach; collaborative strategies; relational capital; organizational efficiencies.

\footnotetext{
'Business Administration, Rey Juan Carlos University, Social Sciences Faculty, Paseo de los Artilleros s/n, 28032 Madrid, Spain, Carmen. E-mail: depablos@urjc.es

${ }^{2}$ Operations Management, ESIC Marketing and Business School,Avda Valdenigrales, s/n, 28222 Pozuelo de Alarcón, Madrid.

E-mail: Ignacio.soret@esic.es

33usiness Administration, UNED, Paseo Juan del Rosal, s/n, 28045 Madrid, Spain. Email: meguilaz@ind.uned.es
}

ISSN: 07 I8-2724. (http://www.jotmi.org)

Journal of Technology Management \& Innovation (c) Universidad Alberto Hurtado, Facultad de Economía y Negocios. 


\section{Introducción}

En la economía globalizada actual, es necesario adaptar los sistemas de innovación empresariales a las características de los mercados demandantes teniendo en cuenta el cambio tecnológico y social. Podemos considerar que nos enfrentamos a una nueva sociedad del conocimiento donde los esfuerzos colaborativos con los clientes a la hora de innovar son imprescindibles. El paradigma de la innovación empresarial ha de basarse en modelos distribuidos $y$, ante todo colaborativos (von Hippel, 2005).

Los usuarios no son simplemente consumidores de productos, donde su comportamiento frente al producto, es pasivo; ahora tienen un papel mucho más activo. La idea tradicional de innovación se basaba fundamentalmente en mantener todo bajo control como un mecanismo de protección de la propiedad intelectual, mientras que la filosofía de innovación actual se centra en la compartición, pensamiento y desarrollo de nuevas fuentes de innovación, como los centros de innovación, las universidades y los individuos.

El principal propósito de las organizaciones tradicionales era el desarrollo de productos o servicios, diferenciados por la generación de ideas exitosas (Von Hippel, 1998). Estas ideas se generaban en las empresas a través de expertos internos que recogían información e ideas en conferencias, proyectos, reuniones, etc. Por tanto, la habilidad de generar ideas de éxito estaba limitada a este perfil profesional. Sin embargo, en un proceso de innovación abierta, el objetivo consiste en buscar las ideas que pueden ser más exitosas donde se encuentren.

Uno de los grandes desafíos de este nuevo paradigma, consiste, por tanto, en identificar, acceder e incorporar el conocimiento necesario para desarrollar un producto o servicio de éxito allá donde se encuentre. La identificación del conocimiento necesario no es una tarea trivial y requiere del conocimiento de lo que está pasando en el sector en el que la organización está inmerso utilizando recursos primarios (investigadores, proveedores, clientes, etc.) y de fuentes secundarias (estudios, estadísticas, etc.).

El objetivo principal de este trabajo consiste en la propuesta de un modelo que permita la medición de conocimiento y la generación de eficiencias organizativas en las iniciativas de innovación abierta con los clientes (lead-user approach, Von Hippel, 2005). Además hemos tratado de analizar otro conjunto de aspectos necesarios para la propuesta del modelo: la identificación, selección y validación de índices que midan capital relacional y eficiencias organizativas. El modelo se compone de dos partes: una que trata de medir el capital relacional, y otra que mide la generación de eficiencias organizativas. El modelo generado es un modelo global que se puede aplicar en cualquier sector.
Una propuesta de valor es una declaración clara de los beneficios que el consumidor final obtiene de utilizar los productos y servicios que la red ofrece. Representa el intento agregado de negocio de todos los nodos de la red o la realización de un intento de negocio de un nodo en la red. La propuesta de valor captura las relaciones entre las ofertas de las empresas y las necesidades inmediatas de los consumidores. Los clientes son el centro de los procesos de innovación donde los diferentes agentes interactúan (Von Hippel, 1986). Deciden como se comportarán los usos de los nuevos productos y servicios (Gambardella, 20I0).

Las organizaciones no se pueden clasificar meramente considerando de forma aislada clientes, proveedores $y$ competidores. De hecho son dos o más de estas dimensiones a la vez. Diferentes actores, empresas, clientes, etc. desarrollan actividades utilizando un conjunto de recursos (Peteraf, 1993). Este modelo propone un conjunto de actores que se conectan por huesos, actividades, enlaces, recursos y lazos.

La ventaja competitiva sostenida se obtiene mediante la posesión de recursos estratégicos que son de valor, raros, difíciles de imitar y no sustituibles (Wernerfelt, 1984) fruto de un conjunto de relaciones que se establece entre oferentes y demandantes de los productos.

El capital relacional expresa el conocimiento y las habilidades que se adquieren en los procesos de comunicación las experiencias de trabajo con los entes con los que se relaciona la empresa (Lesser y Cothrel, 200I). De esta for$\mathrm{ma}$, las actividades sociales juegan un papel importante en la economía hoy, puesto que ofrecen la capacidad de crear y compartir en conocimiento que se necesita para la generación de ventajas competitivas sostenibles (Bueno, 2002).

Coleman $(1990,1998)$ identifica el capital relacional como la capacidad que tienen las personas de trabajar en equipos, de acuerdo a un grupo de reglas y valores compartidos. Sin embargo, para Coleman, el capital relacional, no se encuentra sólo en las relaciones sociales, sino además en la estructura cotidiana donde vivimos las personas. En palabras de este autor, el capital relacional se caracteriza por haber sido creado en la estructura social. Facilita las acciones individuales y colectivas por medio del establecimiento de reglas y sanciones que guían el comportamiento humano. Asimismo, Granovetter (1985) enfatiza que no sólo el grupo de relaciones sociales influyen en las redes, sino además las normas y valores asociados a estas relaciones. La dimensión estructural en el constructo multidimensional del capital relacional, pone de manifiesto la estructura general de relaciones en una empresa. La dimensión relacional trata de argumentar de qué forma las acciones económicas se ven influidas por la calidad de las relaciones (Granovetter, 1992). 
Hatch y Dyer (2004) apoyan la hipótesis de que el capital relacional específico puede crear ventajas competitivas. Debido a que este capital relacional es costoso de imitar, precisamente esas ventajas se pueden sostener (Hatch y Dyer, 2004).

\section{Método}

Previa revisión de la literatura existente, con el fin de obtener información secundaria, tanto interna como externa, e información primaria, se han realizado alrededor de 12 entrevistas con profesionales expertos de diversas empresas que trabajan procesos de innovación. De entre todos ellos, pudimos constituir un panel de 8 expertos.

Con este panel, trabajando en dinámica de grupos y posteriores entrevistas en profundidad con cada uno de los miembros, se llegó a un consenso con respecto a la estructura del modelo más adecuado.

En primer lugar, se trabajó con distintos modelos de medición de capital relacional. Los más significativos son el modelo GLOBAL Scorecard; es preciso anotar que en realidad es más "cuadro de mando integral" (inspirados en el "Balance Scorecard" de Kaplan y Norton) que modelos de conocimiento. Por otra parte, los modelos INTELECT EUROFORUM ESCORIAL y el ampliado a partir de éste, el modelo de los 5 capitales, M5C, son verdaderos modelos de conocimiento.

En segundo lugar, a la hora de medir el impacto del capital relacional en eficiencias empresariales nos hemos focalizado en la metodología de planificación estratégica ITSGA (Andreu et al, 1997), que trata de identificar "acciones genéricas estratégicas" de cuya aplicación pueden distinguirse VCS. Empresas como Sears, Federal Express o TRW han identificado diversas categorías ITSGA para aplicaciones estratégicas como "transacciones con datos de clientes", "desarrollo de nuevos productos" o "establecimiento de diversos acuerdos de colaboración". Además, autores como De Pablos et al. $(2006,2012)$ y ciertos grupos consultores han dado importancia, muy recientemente, en sus trabajos a la identificación de acciones estándar. Es preciso es nuestro caso, siguiendo a sus autores Andreu, Ricart y Valor, razonar por analogía con estos ejemplos para identificar nuestras propias categorías de acciones estratégicas genéricas.Adoptamos, por todo ello, esta metodología que, además, puede integrarse con otras metodologías, por ejemplo con el análisis DAFO como cita Rambla (2006).

Asimismo, se estudió la conveniencia de considerar un conjunto de factores críticos de éxito para la implantación de las prácticas de innovación asociadas a los modelos de lead-user de Von Hippel, previamente citados entre ellos: compromiso de la dirección, liderazgo motivador, equipo polivalente, masa crítica, compromiso de los participantes, sistemas de información, tecnologías de información y comunicación, capacidad de aprendizaje individual y colectivo, capacidad organizativa con orientación a procesos, programas de formación, información de resultados de las acciones de mejora, clima laboral e Iniciativa personal. Estos factores críticos de éxito serían evaluados en un proceso posterior de obtención de información primaria.

Además ambos, las relaciones teóricas en el modelo y los índices propuestos, permiten una comprobación estadística utilizando el modelo de ecuaciones estructurales. De esta forma se asegura la primera hipótesis: el capital relacional en las empresas genera eficiencias en las empresas y particularmente, en las prácticas de innovación centradas en estrategias centradas en el usuario (lead-user).

El modelo de ecuaciones estructurales es apropiado para enfrentarse a este problema debido a su capacidad para integrar de forma directamente observable y medible variables reales y latentes que representan conceptos que no se pueden observar directamente. Las variables latentes en el modelo de ecuaciones estructurales serán: las eficiencias y el capital relacional (observar modelo del cuadro I).A pesar de que podríamos esperar algunas relaciones recíprocas entre las variables, para favorecer la simplicidad en estas primeras etapas de la investigación, presentamos un modelo que contiene sólo relaciones directas y no recíprocas.

Se orientó la constitución de la estructura del modelo a una correspondencia con un Modelo de Ecuaciones Estructurales que permita con posterioridad la validación de dicho modelo, la adecuación de los indicadores propuestos y la corroboración de una teoría que indicara que el incremento del capital relacional en una organización genera eficiencias, por supuesto en el ámbito de acciones innovadoras.

El modelo general de los sistemas de ecuaciones estructurales es un modelo matemático formal, un conjunto de ecuaciones lineales, que comprenden variables directamente observables y medibles (manifiestas) y variables latentes (teóricas) que representan conceptos no observados (Jöreskog, 1993).

Las variables latentes del modelo estructural serían en nuestro caso: Capital Relacional y las eficiencias (Cuadro 4). El modelo MEE establecería que existe una relación directa del capital relacional en la obtención de eficiencias. Además, se incluyen explícitamente errores de medida para cada variable en el proceso de estimación. Las variables medibles u observables serían los indicadores correspondientes del modelo para cada variable latente. 


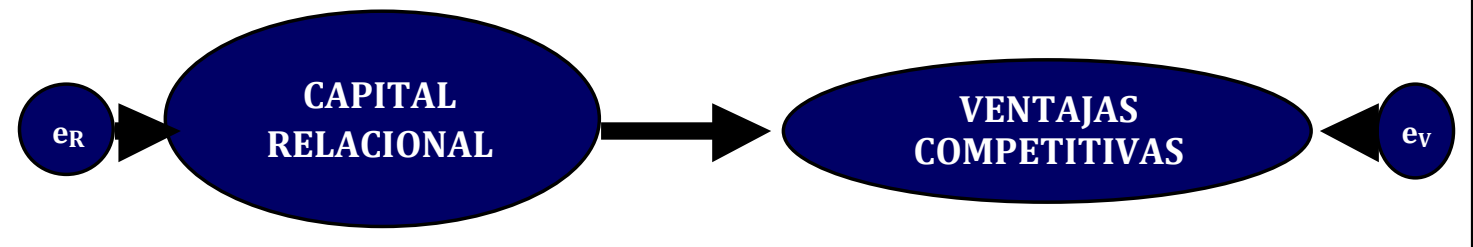

Cuadro I. Modelo de ecuaciones estructurales. Submodelo estructural.

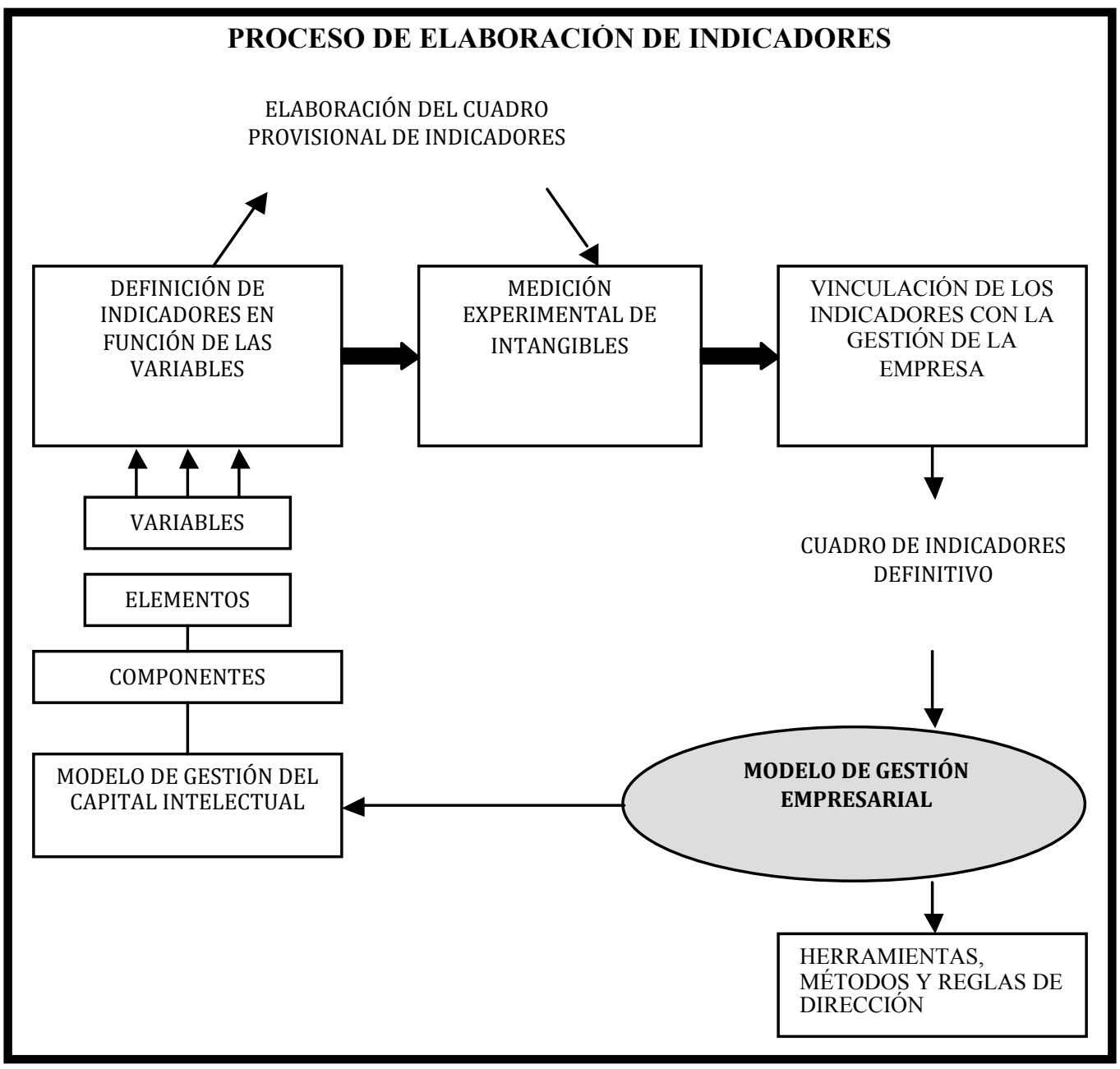

Cuadro 2. Elaboración de indicadores. Fuente: Rodríguez (2003).

ISSN: 07 I8-2724. (http://www.jotmi.org)

Journal of Technology Management \& Innovation (c) Universidad Alberto Hurtado, Facultad de Economía y Negocios. 
Indicadores de capital relacional y eficiencias empresariales Este trabajo tiene como objetivo principal la propuesta de un modelo que permita la medición de conocimiento y la generación de eficiencias organizativas en las iniciativas de innovación abierta con los clientes Sin embargo, cabe destacar otros objetivos específicos, siguiendo la dinámica de trabajo con el equipo de expertos, como son los siguientes:

- Identificación, selección y validación de indicadores de capital relacional y de eficiencias.

- Posibilidad de una metodología de validación del modelo de medición propuesto y del grado de adecuación de los indicadores.

El modelo de medición que proponemos, contiene dos grandes bloques: uno de conocimiento o capital relacional y otro de eficiencias. Para desarrollar ambos bloques, como ya se ha dicho, revisamos los modelos existentes y la metodología de planificación estratégica ITSGA (Acciones estratégicas genéricas basadas en las tecnologías de la información).

Así pues, proponemos nuestro modelo de medición de capital relacional, CR, y Eficiencias. En lo que se refiere a eficiencias, consideramos 5 categorías de acciones estándar:"pro- ducto", "clientes”, "canales", "proveedores” y "de carácter general". Por analogía, denominamos al segundo gran bloque de EFICIENCIAS, EFINNOVA, es decir:"acciones genéricas estratégicas basadas en prácticas de innovación centradas en el usuario".

Para la elaboración de indicadores hemos seguido las observaciones y propuestas del panel de expertos junto con otras referencias bibliográficas $y$, especialmente, hemos tomado en cuenta el proceso de elaboración de indicadores de capital intelectual de acuerdo con Rodríguez (2003), Bueno, Rodríguez y Salmador (2003) e IADE-CIC (2003). En dicho proceso puede emplearse tanto un método deductivo como inductivo. Siguiendo con Rodríguez (2003), la complementariedad de estas metodologías es notoria, ya que la evidencia empírica se encuentra en el origen del método inductivo y en el término de las deducciones lógicas del método deductivo. Por esta razón, resulta aconsejable la combinación de ambos métodos en la elaboración de indicadores de capital relacional, así como para las eficiencias empresariales. Los pasos se muestran en el cuadro 2.

Una vez identificados los elementos de cada componente del modelo de medición y sus variables más representativas, se inicia el proceso de elaboración de indicadores. Las

\begin{tabular}{|c|c|}
\hline $\begin{array}{l}\text { VARIABLES DEL MOD- } \\
\text { ELO DE MEDICIÓN }\end{array}$ & \\
\hline $\begin{array}{l}\text { BLOQUE I: CAPITAL } \\
\text { RELACIONAL }\end{array}$ & $\begin{array}{ll}\text { - } & \text { Mejora en el conocimiento de clientes } \\
\text { - } & \text { Iniciativas de mejora de satisfacción de clientes } \\
\text { - } & \text { Eficiencia en acuerdos de colaboración } \\
\text { - } & \text { Participación en prácticas con agentes de la cadena de suministro } \\
\text { - } & \text { Incremento de satisfacción de los stakeholders } \\
\text { - } & \text { Eficiencia de CRM-Coooperativo } \\
\text { Eficiencia de B2B, B2C y otras }\end{array}$ \\
\hline $\begin{array}{l}\text { BLOQUE II: EFI- } \\
\text { CIENCIAS }\end{array}$ & 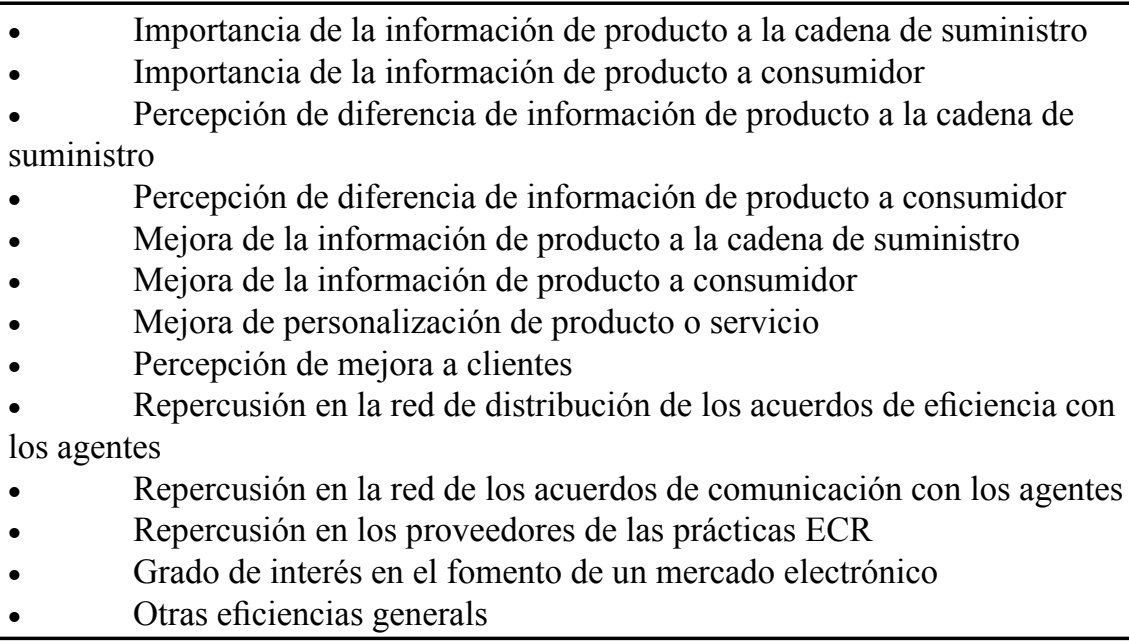 \\
\hline
\end{tabular}

Cuadro 3.Variables del modelo de medición.

ISSN: 07 I8-2724. (http://www.jotmi.org)

Journal of Technology Management \& Innovation (c) Universidad Alberto Hurtado, Facultad de Economía y Negocios. 
características de los mismos vendrán determinadas por la naturaleza de las variables a medir. Así se elaborará un cuadro provisional de indicadores que recogerá los criterios de medida para las variables representativas de los elementos del modelo. Será después de una medición experimental cuando puedan perfeccionarse los indicadores diseñados y establecer criterios de agregación de los mismos. Finalmente, se vincularán con el modelo de gestión de la empresa. Las variables consideradas para cada uno de los bloques del modelo se presentan en el cuadro siguiente.

\section{El modelo}

Cada uno de los 6 bloques propuestos (I de CR y 5 de EFINNOVA) se subdivide en elementos que suman en total 36; cada elemento puede subdividirse a su vez en otros subelementos, consiguiendo así un total de 158 indicadores - variables que pueden ser medidas mediante "percepciones de mejora" (con una escala de Likert, por ejemplo), constituyendo así nuestra propuesta final de modelo de medición de la innovación centrada en prácticas con el usuario y de eficiencias empresariales (Cuadro 4).

La estructura de nuestro modelo se adapta al modelo científico, hipotético deductivo, con el fin, en un futuro, de enunciar ciertas proposiciones que podrían corroborar una teoría. Se partiría de un conocimiento de fondo no problemático para enunciar ciertas hipótesis teóricas, de las que podrían deducirse otras básicas contrastables estadísticamente. En nuestro caso, modelo científico, modelo de ecuaciones estructurales y modelo de medición propuesto sintonizan perfectamente a tal efecto.
En definitiva, los indicadores de prácticas de innovación Capital relacional junto a los indicadores de EFINNOVA, acciones estratégicas basada en la adopción de prácticas de innovación centradas en el cliente, pueden expresarse en los siguientes cuadros 5 y 6 .

\section{Discusión}

En este trabajo hemos tratado de proponer un modelo que permita la medición del impacto de las prácticas de innovación empresarial que se centran en el cliente y la generación de eficiencias organizativas.

Para la medición del capital relacional, hemos optado por el modelo INTELECT para aplicarlo en el ámbito de la innovación producida por prácticas colaborativas con los clientes, por las siguientes razones: es de fácil comprensión y, según Bueno, Rodríguez y Salmador (2003), abierto, flexible y con validez universal y, por otra parte, es el más extendido y operativo en España (por ejemplo, ha sido considerado en organizaciones como BBVA, Pricewaterhouse\&Coopers, Telefónica I+D, Correos y Telégrafos, Ayuda en Acción, Zara, Seguridad Social, Idom,...).

En cuanto a la medición del impacto de las estrategias de innovación centradas en los clientes en las eficiencias organizativas, nos hemos focalizado en la metodología de planificación estratégica ITSGA, que trata de identificar "acciones genéricas estratégicas" de cuya aplicación pueden distinguirse ventajas competitivas sostenibles para las organizaciones. Empresas como Sears, Federal Express o TRW han identificado diversas categorías ITSGA.

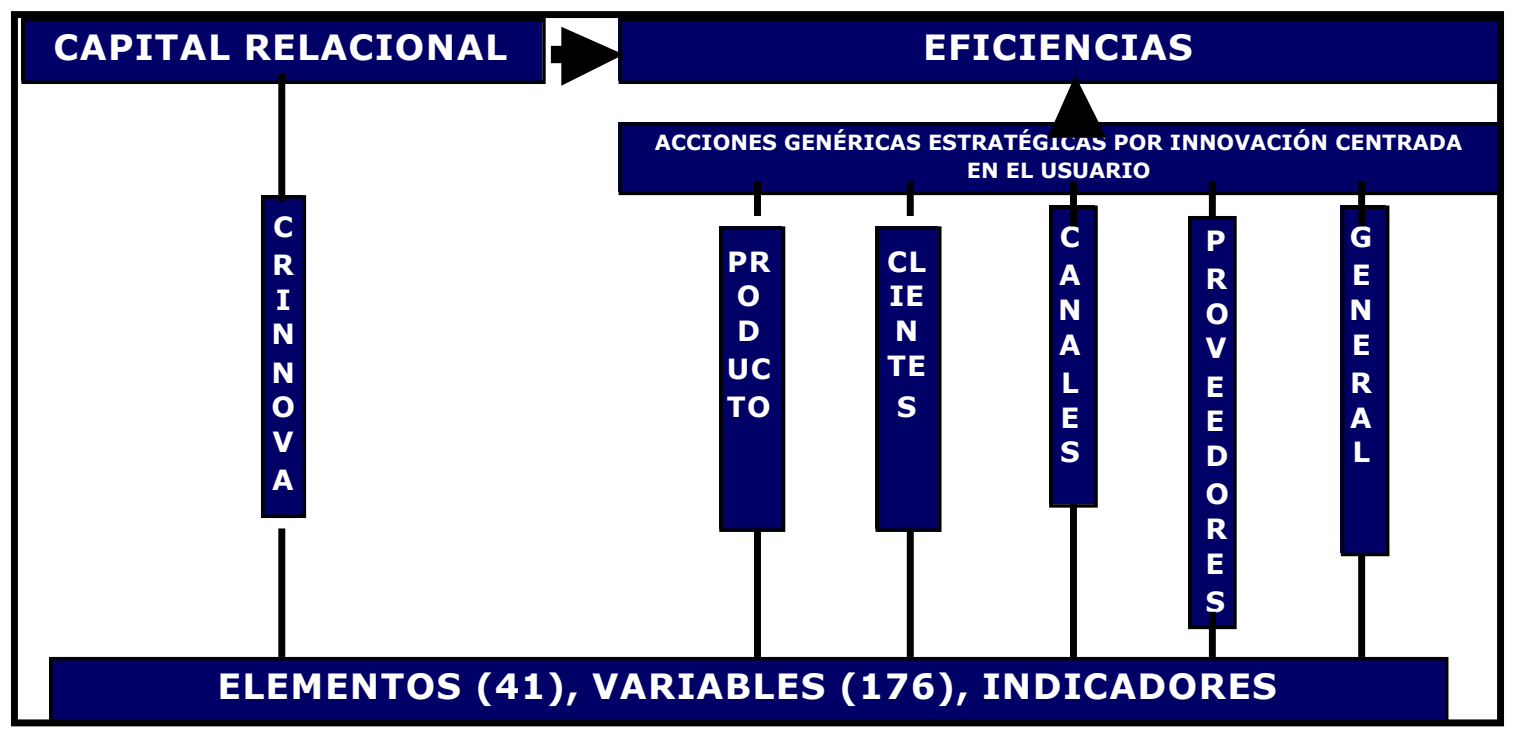

Cuadro 4. Modelo de medición de Cl yVCS en el ámbito ECR (adaptado de Soret, 2007).

ISSN: 07 I8-2724. (http://www.jotmi.org)

Journal of Technology Management \& Innovation (c) Universidad Alberto Hurtado, Facultad de Economía y Negocios. 


\section{INDICADORES del MODELO EFININNOVA}

\section{CAPITAL RELACIONAL}

\begin{tabular}{|c|c|}
\hline $\begin{array}{l}1 . \\
\bullet \\
\bullet \\
\bullet \\
\bullet \\
\bullet\end{array}$ & $\begin{array}{l}\text { BBDD de clientes: } \\
\text { Nuevos clientes } \\
\text { Fidelización } \\
\text { Up-selling } \\
\text { Cross-selling }\end{array}$ \\
\hline 2. & $\begin{array}{l}\text { Mejora de satisfacción de } \\
\text { clientes }\end{array}$ \\
\hline 3. & Lealtad de clientes \\
\hline 4. & $\begin{array}{l}\text { Incremento de acuerdos de } \\
\text { colaboración }\end{array}$ \\
\hline 5. & $\begin{array}{l}\text { MP: } \\
\text { AFM, OER, CRP, ... }\end{array}$ \\
\hline 6. & $\begin{array}{l}\text { Existencia de indicadores de } \\
\text { satisfacción de stakeholders }\end{array}$ \\
\hline 7. & EIP (Portales de información) \\
\hline 8. & CRM cooperativo: SFA, ... \\
\hline 9. & $\mathrm{~B} 2 \mathrm{~B}, \mathrm{~B} 2 \mathrm{C}, \ldots$ \\
\hline
\end{tabular}

Cuadro 5. Indicadores del modelo efinnova.

INDICADORES DE OBTENCIÓN DE EFICIENCIAS

EFICIENCIAS para los productos

Mejorar y/o incrementar la información del producto

Personalización de producto (en sí mismo, a través de envase y/o embalaje, etiquetado, ...)

EFICIENCIAS para los consumidores

Mejora de precios

Mejora de surtido

Mejora de promociones

Creación de portales de información EPI,s

EFICIENCIAS para los canales de distribución

Acuerdos de eficiencia

Acuerdos de comunicación con TIC

EFICIENCIAS para los proveedores

- CRP

- $\quad$ CPFR

- AFM

- OER

EFICIENCIAS generales

- Mejores prácticas de carácter general

- $\quad$ Fomentar un mercado electrónico

Cuadro 6. Indicadores de obtención de ventajas competitivas.

ISSN: 07 I8-2724. (http://www.jotmi.org)

Journal of Technology Management \& Innovation (c) Universidad Alberto Hurtado, Facultad de Economía y Negocios. 
Los indicadores de generación de capital relacional y de eficiencias organizativas son de difícil cuantificación. Por este motivo, sugerimos la utilización de una medida cualitativa: la percepción.

El modelo propuesto es un modelo global no aplicado a ningún sector en particular aún. Sin embargo, dadas las características de los elementos del mismo, puede ser particularizado a distintos segmentos de interés y a distintas empresas. En el modelo propuesto se integran, de forma novedosa, el capital relacional y un conjunto de indicadores de eficiencias organizativas.

Como futuras líneas de investigación, creemos que merece la pena aplicar el modelo a diferentes sectores. También parece conveniente incluir una dimensión temporal en el modelo, ya que el grado de implantación y despliegue de las prácticas de innovación que se centran en los clientes suelen ser a medio o largo plazo. Por último, estimamos que sería necesario analizar, mediante entrevistas en profundidad, las causas de abandono de ciertos proyectos piloto e identificar así factores inhibidores de la adopción de prácticas de innovación centradas en los clientes.

\section{Referencias}

ANDREU, R., Ricart, J.E.,Valor, J. (1997). La organización en la era de la información, Madrid. McGraw-Hill.

BUENO, E., Salmador, M.P., Rodríguez, O., Martín de Castro, G. (2004). Fundamentación bio-mimética y la explicación del valor en el modelo Intellectus de capital intelectual: evidencia empírica de la existencia de una posible "neurona creativa", Congreso ACEDE, Murcia.

BUENO, E. (2002). El capital social en el nuevo enfoque del capital intelectual de las organizaciones. Revista de Psicología del Trabajo y de las Organizaciones, I2(I), I34-I46.

BUENO, E. ( 1998). El capital intangible como clave estratégica en la competencia actual, Boletín de estudios económicos, vol. LIII.

COLEMAN, J.S. (1998). Social capital in the creation of human capital. American Journal of Sociology, 94, 95- 120.

COLEMAN, J.S. (1990). Foundation of social theory. Cambridge: Harvard University Press.

CHRISTENSEN, C. (2003). The innovators dilemma: the revolutionary book that will change the way you do business. Cambridge: Collins Business Essentials.
DE PABLOS, C., López-Hermoso, J.J., Martín-Romo, S., Medina, S., Montero, A., Nájera, J. J. (2006). Dirección y gestión de los sistemas de información en la empresa, Madrid: ESIC.

DE PABLOS, C., López-Hermoso, J.J., Martín-Romo, S., Medina, S. (20I2). Organización y transformación de los Sistemas de Información en la Empresa. Madrid: ESIC.

FJELDSTAD, O., Stabell, Ch. (1998). Configuring value for competitive advantage: on chains, shops and networks. Strategic Management Journal, 19(3), 33-44.

FREEEMAN, C. (1988). Japan: a new national system of innovation. In. G. Dosi et al. Technical change and Economic Theory, London: Pinter.

GAMBARDELLA, A. (20I0). Innovation and developments: myths and realities, Conference cycle, Managing the crisis: business strategies to face the economic crisis, Fundación Ramón Areces, Madrid, 3 I st May.

GRANOVETTER, M.S. (1992). Problems of explanation in economic sociology. En N. Nohria y R. Eccles (Eds.), Networks and organizations: Structure, form and action. Boston: Harvard Business School Press.

GRANT, R.M. (1998). Contemporary Strategy Analysis: Concepts, Techniques, and Applications (3rd edn). Cambridge, MA: Blackwell Business Press.

EVANGELIDIS, A. (2005). FRAMES - A risk assessment framework for e-services. Electronic Journal of e-government, 3(I), September.

HATCH, N.W., Dyer, J.H. (2004). Human capital and learning as a source of sustainable competitive advantage. Strategic Management Journal, 25, I.I55-I.I78.

JÖRESKOG, K. G. (1993). Modelado de ecuaciones estructurales. Guipúzcoa: Euskadiko,.

KAVAN, B., O'Hara, M., Patterson, E., Bostrom, R. (1999). Excellence in Client/Server Information System Implementations: understanding the STS Connection. Management Decision, 37(3), 295-30I.

LESSER, E., Cothrel, J. (200 I). Fast friends: virtuality and social capital. Knowledge Directions, spring-summer, 66-79.

LUNDVALL, B.A. (1992). National Systems of Innovation. London: Pinter.

PETERAF, M.A. (1993). The Cornerstones of Competitive Advantage: A Resource-Based View. Strategic Management Journal, I4(99), I79-192

ISSN: 07 I 8-2724. (http://www.jotmi.org) 
PORTER, M.E. (I990).The competitive advantage of Nations, London: MacMillan.

RAMBLA, D. (2006). ITSGA: acciones estándar a través de cuya aplicación pueden conseguirse ventajas competitivas sostenibles, www.danielrambla.com.

RODRÍGUEZ, L. (2003). Valoración y gestión de intangibles: aproximación teórica y estudio empírico. Informe. Universidad de Salamanca.

VON HIPPEL, E. (1986). Lead users: a source of novel product concept. Management Science, 32(7), 79I-805.

VON HIPPEL, E. (2005). Democratizing Innovation. Cambridge, MA: MIT Press.

VON HIPPEL, E. (1998). The sources of innovation. New York: Oxford University Press.

WERNERFELT, B. (1984). The Resource-Based View of the Firm. Strategic Management Journal 5(2), I7I-I80. 